\title{
Outcome of Surgical Treatment for Arterial Thoracic Outlet Syndrome
}

\author{
B.Velladuraichi ${ }^{1}$, S.Jeyakumar ${ }^{2}$, P.Ilayakumar ${ }^{3}$, N.Sritharan ${ }^{4}$ \\ 1, 2, 3, 4 (Institute of Vascular Surgery, Madras Medical College, India)
}

\begin{abstract}
:
Objective: The purpose of this study was to review our operative experience and to assess the symptomatic outcome of patients with arterial Thoracic outlet syndrome who underwent decompression of the thoracic outlet. Methods: This is a prospective study of patients with arterial thoracic outlet syndrome treated with cervical rib excision and scalenectomy with or without Subclavian artery revascularization at Institute of Vascular Surgery, Madras Medical College, Chennai.

Results: Patients were a mean age of 37.8 years. $36.2 \%(n=21)$ of patients had only compression with no arterial lesion. Subclavian artery aneurysm (44.8\%) was the commonest arterial lesion when compared to dilatation (13.7\%) and occlusion (13.7\%). All patients underwent thoracic outlet decompressin of which there were $93.4 \%(n=54)$ Cervical rib, $3.4 \%(n=2)$ first rib excision and $3.4 \%(n=2)$ soft tissue resection. Subclavian artery reconstruction was performed in $63.7 \%(n=37)$ of patients. One patient $(1.7 \%)$ who had advanced ischemia underwent above elbow amputation in the postoperative period. Subclavian artery patency was 100\% with no dilatation, aneurysm or embolism.

Conclusion: In our experience, cervical rib excision with scalenectomy through supraclavicular approach is an effective procedure with less morbidity and mortality.
\end{abstract}

Keywords: cervical rib, endartrectomy, interposition graft, subclavian aneurysm, Thoracic outlet syndrome.

\section{Introduction}

The diagnosis and treatment for thoracic outlet syndrome pose a significant challenge to vascular surgeons. Thoracic outlet syndrome patients have a constellation of symptoms that are presumed to result from compression of the neurovascular structures supplying the upper extremity [1] The commonest form of TOS is neurogenic type. The arterial Thoracic Outlet Syndrome (aTOS) forms less than 1\% of cases, but the morbidity and mortality is severe that warrants immediate attention and management. The syndrome is caused by the cervical rib or anomalous first rib eliminating the space under the subclavian artery [2,3]. This external compression results in repeated trauma in the intima of subclavian artery, resulting in subclavian stenosis, thrombosis, aneurysm formation with mural thrombus and distal embolisation. Arterial complications of thoracic outlet syndrome are unusual. However, they are potentially much more serious than the more common neurologic manifestations, and in some cases they may lead to loss of limb. The presence of symptoms varies according to the subclavian artery disease status. Symptoms are acute upper limb ischemia like parasthesia, pain, pallor, coldness, digital ischemia and weakness or chronic ischemia like arm claudication.

Surgery is the only option in arterial Thoracic outlet syndrome. The surgical armamentarium contains scalenectomy with or without rib excision, trans axillary first rib excision, resection of any constraining band or soft tissue, in addition to the management of Subclavian artery (SCA) disease such as thrombectomy, Endartrectomy and aneurysm resection with bypass. Cervical rib excision with scalenectomy is sufficient to relieve the symptoms of TOS without excision of the first rib in most of the cases those who presented early. Those who are presenting late with distal ischemia, the subclavian artery require reconstruction as well. Successful outcome after TOS is determined by relief of symptoms, patency of arterial bypasses, avoidance of recurrence and limb salvage.

This study summarizes the nature of the arterial lesion and outcome of 58 patients who underwent surgical decompression for arterial TOS at our Institute.

\subsection{Patients}

\section{Materials and methods}

This prospective study includes analysis of 58 patients who were admitted at Institute of Vascular Surgery, Madras Medical College, Chennai-3, with arterial lesions in the upper limb due to Thoracic outlet syndrome from August 2010 to April 2017.Patients with atherosclerotic lesion, Neurogenic TOS and primary Raynaud's phenomenon were excluded from this study. 


\subsection{Methods}

\subsubsection{Evaluation}

On admission all patients are evaluated for the emergency nature of the disease by recording thorough history and physical examination like supraclavicular fullness (Fig.1.A) and pulse examination. If signs of distal embolisation (Fig.1.B) present to confirm the level and severity of ischemia, we measure the brachial, radial and ulnar artery pressure using hand Doppler. A cervical spine x ray (Fig.1.C) was obtained as a means of identifying certain anatomic abnormalities like cervical rib. No additional diagnostic studies were routinely performed in acute cases. After obtaining anaesthetic assessment, we proceed with thoracic outlet decompression and thrombectomy as early as possible. In chronic limb ischemia, the patient was evaluated by Duplex scan and computed Tomography (Fig.1.D, E) in case of distal occlusion. If patients with arterial TOS with no cervical rib, we performed Magnetic resonance imaging to identify the soft tissue lesion.

\subsubsection{Surgery}

All patients were approached through supraclavicular route. After incising the skin, platysma scalene pad of fat was mobilized superolaterally and phrenic nerve was preserved. Anterior scalene muscle was identified and divided. Subclavian artery control was taken. In all cases cervical rib was excised along with the soft tissue structures compressing the artery. According to the type of lesion and the level of embolisation, endartrectomy with SCA suturing with either thrombectomy through SCA or Brachial artery was performed. Patients with distal occlusion bypass were performed using synthetic or venous graft.

\subsection{Outcome}

All patients were examined clinically to assess the postoperative improvement. Clinical assessment of the results for arterial reconstruction included examination for any evidence of digital emboli or ischemia, bilateral upper extremity blood pressures and Duplex scan. All patients were followed up monthly up to first three months, then once in three months in first year and once in a year till date. On selection each case is studied in detail and the data was collected by using the proforma. The data obtained from proforma are entered in computer in excel format. The data is presented in appropriate tables, charts and graphs Analysis of data was done and statistically interpreted.

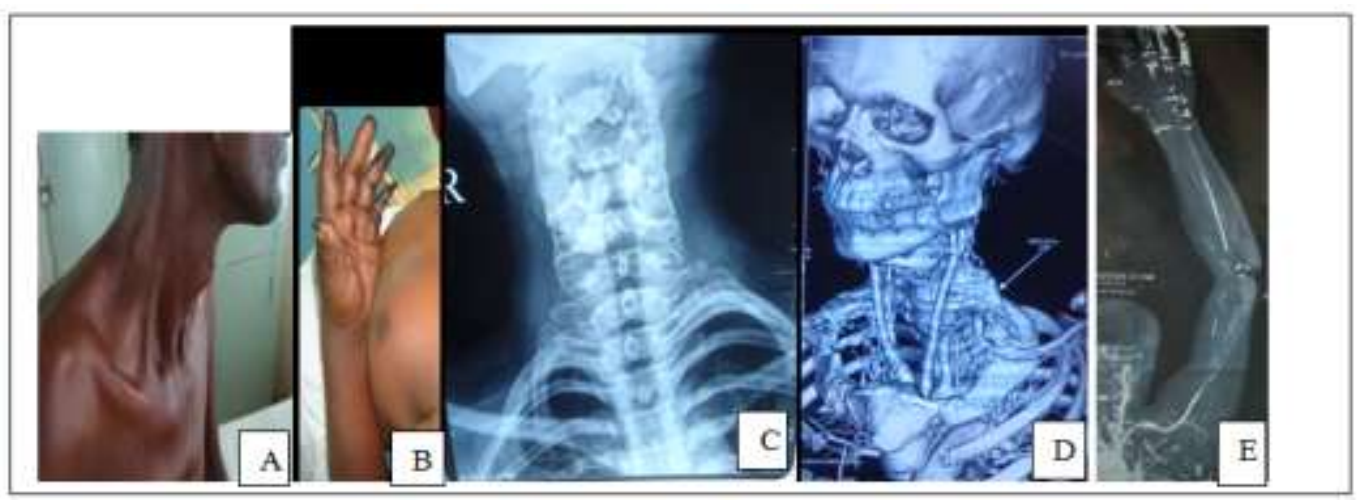

Fig.1.Arterial TOS: Clinical finding and investigations. A) Supraclavicular fullness, B) Digital gangrene, C) Xray neck showing bilateral cervical rib, D,E) Computed tomography showing post stenotic dilatation

\subsection{Clinical presentation}

\section{Results}

58 cases who underwent surgical decompression were studied in detail. Patients were a mean age of years, range $13-65$ years) and $58.6 \%(n=39)$ male, $41.3 \%(n=19)$ female (Fig: 2$)$.

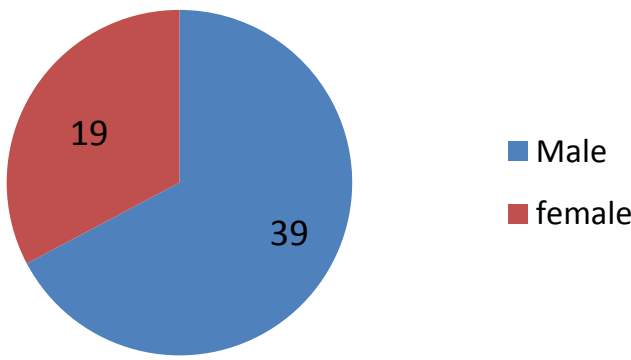

Fig:2 -Sex Distribution 
Usually arterial TOS is recognized only after the occurrence of distal thrombo embolism causing distal ischemic changes. Associated distal arterial lesion is found in 51.7\% of cases. The commonest distal site of occlusion is brachial artery (56.6\%) followed by axillary (26.6\%) and forearm arteries (16.6\%). A cervical rib was identified by pre operative chest X-ray in $54(93.1 \%)$ cases, first rib in $2(3.4 \%)$ cases. $2(3.4 \%)$ cases with no bony abnormalities had Magnetic Resonance Imaging which showed soft tissue compressing the SCA (Fig: 3).majority of patients $62.06 \%$ were in Scher III, $32.7 \%$ of patients were in sher I and $5.17 \%$ of patients were in Scher II

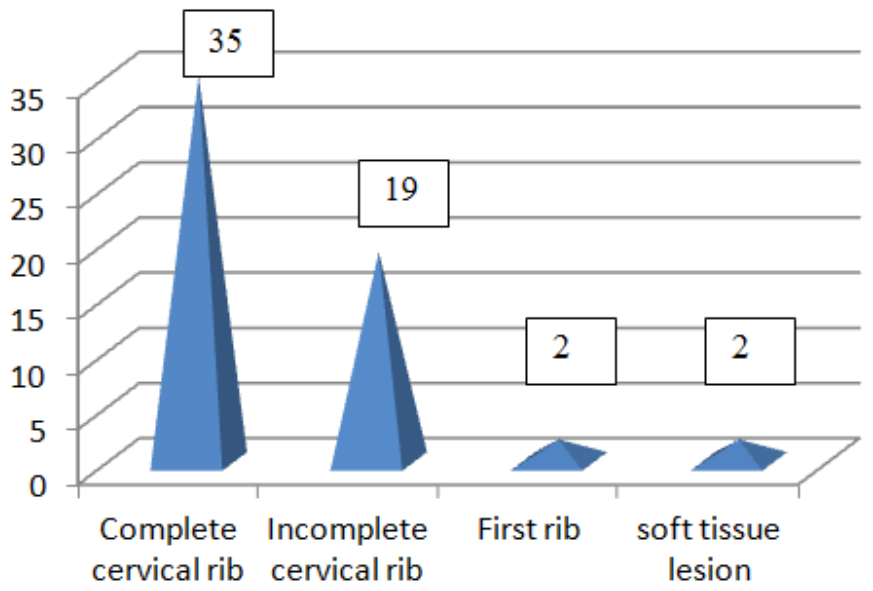

- Series1

Fig: 3-Compressing agents

\subsection{Surgical treatment}

All patients underwent thoracic outlet decompression through supraclavicular approach. The arterial lesions found in Subclavian artery were dilatation (13.7\%) (Fig.4A), aneurysm (44.8\%) (Fig.4B), and occlusion $(13.7 \%)$. Thrombus (Fig.4C) is seen in 34(62.06\%) cases, most commonly associated with aneurismal lesion of SCA. Cervical rib excision (Fig.4F) with anterior scalenectomy in $93.1 \%$ of patients, first rib in $3.4 \%$ patients and anterior scalenectomy and soft tissue resection in $3.4 \%$ patients. $63.7 \%$ of patients had SCA intervention. Endartrectomy (Fig.4D, E) was performed in $6.9 \%$ of patients. Endartrectomy with thrombectomy through SCA was done in $29.31 \%$ of patients. $17.24 \%$ of patients underwent concomitant brachial thrombectomy at the time of endarterctomy. Direct SCA reconstruction was performed in $10.3 \%$ of patients with interposition bypass grafting using synthetic graft Dilatation (13.7\%) (Fig.4G) in 4 patients and reverse saphenous vein in 2 patients. $36.2 \%$ of patients had no SCA reconstruction.

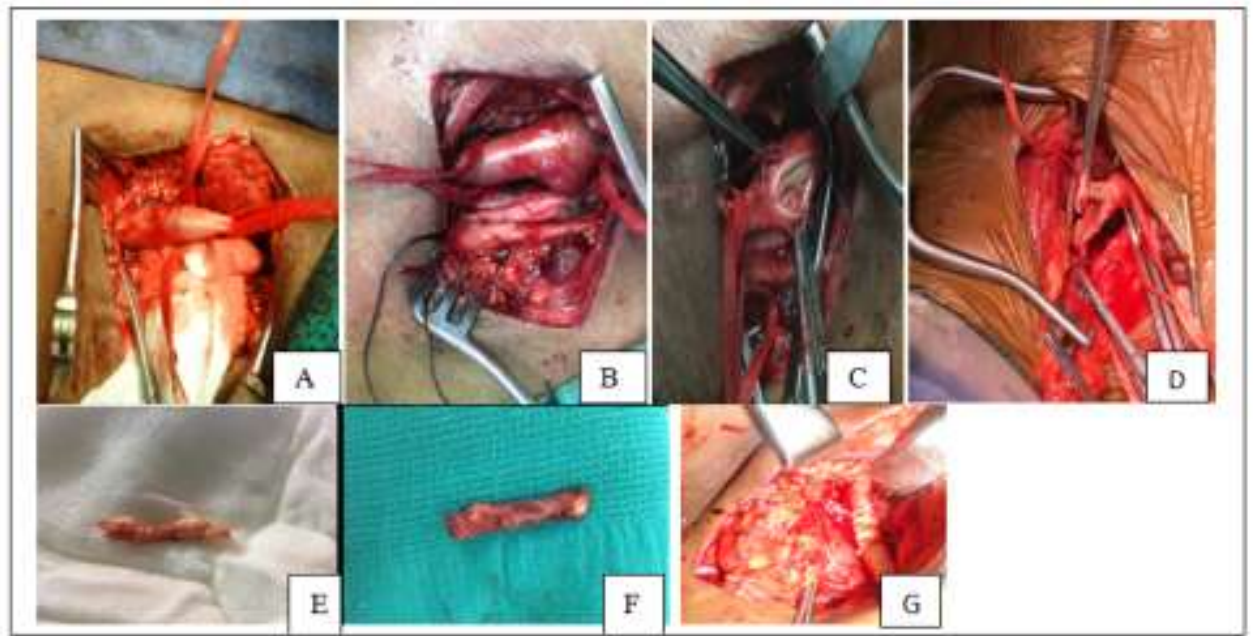

Fig.4.Subclavian artery reconstruction and cervical rib excision, A) Subclavian artery dilatation, B) Subclavian artery aneurysm, C) Subclavian artery thrombus D,E)Endartrectomy, F)Excised cervical rib, G) Interposition graft using ringed PTFE graft.

(PTFE-poly tetra fluro ethylene) 


\subsection{Outcome}

There was no peri operative and postoperative mortality. Two (3.4\%) patients had surgical site hematoma. One (1.7\%) patient underwent above elbow amputation, who had advanced ischemia at the time of presentation. Overall limb salvage rate is $98.2 \%$. There were two (3.4\%) finger amputation and eight $(13.7 \%)$ distal phalangeal amputations (Fig: 5).

\subsection{Clinical follow up}

During the 7 years follow up only two patient developed recurrent upper limb pain. $86.4 \%(n=32)$ of patients who had SCA intervention had palpable pulse and $13.5 \%(n=5)$ patients with distal forearm lesion had pressure improvement.

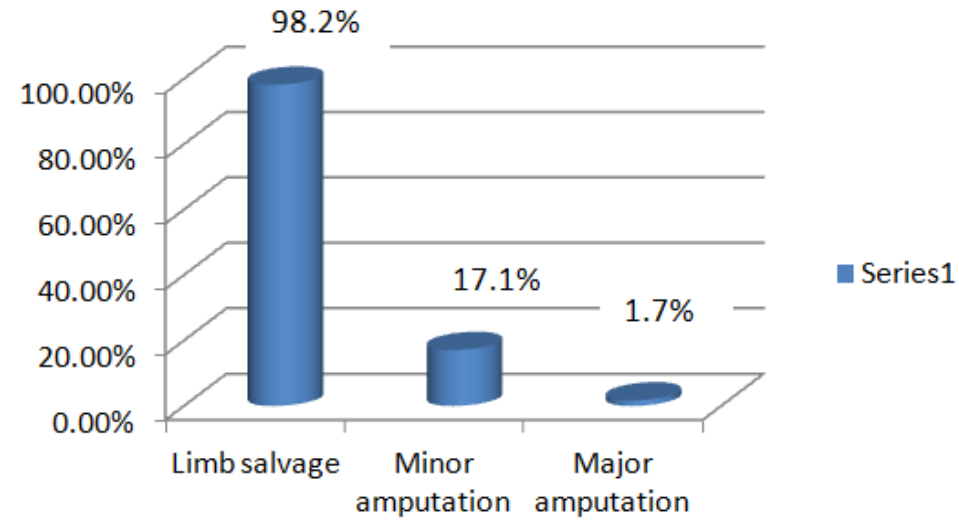

Fig: 5-Outcome

\section{Discussion}

Although the subclavian artery can be compressed in the region of the thoracic outlet by a variety of osseous, muscular, and ligamentous structures, the overwhelming majority of arterial complications are produced by complete cervical ribs. Although cervical ribs are estimated to occur in $0.5 \%$ of the population [4], most are asymptomatic and arterial lesions are uncommon. Early diagnosis and aggressive treatment of arterial complications of thoracic outlet syndrome are indicated to prevent disabling upper extremity ischemia and even digital gangrene.

In our study, Mean age is 37 years [5, 6-8], 58.6\% were male, in contrast to common belief that females are more commonly affected. In a report by Adson [9] it was $72 \%$ were women and $23 \%$ were men. In other studies also there is female preponderance attributed to the presence of cervical ribs more in females than in males. But the presence of cervical rib does not mean TOS and so the increased number of cervical ribs in females does not means increased number of arterial TOS in females. We believe that resection of the cervical rib and anterior scalene muscle is appropriate for relief of symptoms in most patients. Davies et al [10] previously studied excision of the cervical rib alone, without removal of the first rib. In a study of 58 patients with TOS, 22 patients were found to have cervical ribs. In their population, 15 patients received cervical rib resection alone and were cured of all symptoms. However, two patients who had cervical ribs previously removed had continued symptoms and eventually required a first rib resection for further symptomatic improvement. Toso et al [11] also found that patients who had only the cervical rib removed without the first rib removed continued to have symptoms after operation. We found that removing the cervical rib and anterior scalenectomy is an effective treatment. We found no evidence of increased morbidity or mortality in patients who received resections of the cervical rib. We have found that aggressive treatment may be necessary to return arterial flow and relieve arterial compression, which accounts for our high cure rate. Because subclavian aneurysm formation and thrombosis may result from prolonged arterial compression by a cervical rib, further intervention in addition to cervical rib resection in the form of PTFE or saphenous vein grafts is often required $[12,13]$. In our population, 26 patients were presented with a subclavian artery aneurysm complicated by thrombosis and embolization to the arm. These patients required placement of a subclavian-axillary interposition bypass graft with PTFE (four patients) or reverse saphenous vein (2 patients) and an embolectomy of the axillary, brachial, radial, and ulnar artery in order to restore flow to the upper extremity. Supraclavicular approach is best for cervical rib excision and for gaining access to the subclavian artery [14, 15, 16-18]. A combined supraclavicular and infraclavicular approach has also been suggested to allow for access to both the subclavian and axillary arteries, but four of our patients who had arterial reconstruction required a dual approach [19-23]. Immediate post operative period only two patients had hematoma. During follow up period two patients 
with occlusion at forearm had recurrent pain but angiogram showed no dilatation or thrombus proximally. Published series report complete relief of symptoms in more than $90 \%$ of patients [24, 25-27].

Bypass patency in our study is $100 \%$. In other series bypass patency is reported in the range of 90 $100 \%$ depending patency of the outflow [26, 28]. Limb salvage in Scher I and II is 100\%, distal embolization may result in finger and digital amputation in scher III but arm amputation is uncommon [28]. In our series Limb savage in Scher I and II is $100 \%$ and scher III is $97.4 \%$. All other patients are asymptomatic. None of our patients had retrograde thrombosis of vertebral artery which has also been reported as a complication of thoracic outlet compression [29, 30-34].

\section{Conclusion}

This study confirms that when thromboembolic events are present, Surgery is the only option all thoracic outlet syndrome cases. However early diagnosis before thromboembolism is the goal. We also conclude in patients with arterial TOS due to cervical rib, supraclavicular approach and cervical rib excision with anterior scalenectomy with or without thrombectomy is an effective procedure.

\section{References}

[1]. Urschel, HC and Razzuk, MA. Management of the thoracic outlet syndrome. N Engl J Med. 1972; 286: 1140-1143

[2]. Maru S, et al: Thoracic outlet syndrome in children and young adults. Eur J Vasc Endovasc Surg 38:560-564, 2009.

[3]. Chang K, et al: Spectrum of thoracic outlet syndrome presentation in adolescents. Arch Surg 146:1383-1387, 2011.

[4]. White JC, Poppel MH, Adams R. Congenital malformations of the first thoracic rib. Surg Gynecol Obstet $1945 ; 81643.59$.

[5]. Chang K, et al: Spectrum of thoracic outlet syndrome presentation in adolescents. Arch Surg 146:1383-1387, 2011

[6]. Fields W, et al: Thoracic outlet syndrome: review and reference to stroke in a major league pitcher. Am J Neuroradiol 146:809-814, 1986.

[7]. Gooneratne IK, et al: Pearls and oysters: distal subclavian artery. A source of cerebral embolism. Neurology 73:e11-e12, 2009.

[8]. Naz I, et al: Cerebral embolism: distal subclavian disease as a rare etiology. J Pak Med Assoc 56:186-188, 2006.

[9]. Adson AW: Surgical treatment for symptoms produced by cervical ribs and the scalenus anticus muscle. Surg Gynecol Obstet 85:687-700, 1947

[10]. Davies AH, Walton J, Stuart E, Morris PJ. Surgical management of the thoracic outlet compression syndrome. Br J Surg 1991; 78:1193-5.

[11]. Toso C, Robert J, Berney T, Pugin F, Spiliopoulos A. Thoracic outlet syndrome: influence of personal history and surgical technique on longterm results. Eur J Cardiothorac Surg 1999; 16:44-7.

[12]. Rob CG, Standeven A. Arterial occlusion complicating thoracic outlet compression syndrome. Br Med J 1958; 2:709-12.

[13]. Gelabert HA, Machleder HI. Diagnosis and management of arterial compression at the thoracic outlet. Ann Vasc Surg 1997; 11:359-6614.

[14]. Nehler MR, et al: Upper extremity ischemia from subclavian artery aneurysm caused by bony abnormalities of the thoracic outlet. Arch Surg 132:527-532, 1997.

[15]. Lee TS, et al: Cerebral embolic stroke and arm ischemia in a teenager with arterial thoracic outlet syndrome: a case report. Vasc Endovascular Surg 41:254-257, 2007.

[16]. Adson AW: Surgical treatment for symptoms produced by cervical ribs and the scalenus anticus muscle. Surg Gynecol Obstet $85: 687-700,1947$

[17]. Hugl B, et al: Unusual etiology of upper extremity ischemia in a sclero- derma patient: thoracic outlet syndrome with arterial embolization. J Vasc Surg 45:1259-1261, 2007.

[18]. Fields W, et al: Thoracic outlet syndrome: review and reference to stroke in a major league pitcher. Am J Neuroradiol 146:809-814, 1986.

[19]. Davidovic LB, Koncar IB, Pejkic SD, Kuzmanovic IB. Arterial complications of thoracic outlet syndrome. Am Surg 2009; 75:2359.

[20]. Dovgan PS, Edwards JD, Ayoub NT, Thorpe P, Agrawal DK. Arterial embolism from anatomical variation at the thoracic outlet. Clin Anat 1995; 8:222-6.

[21]. Sanders RJ, Hammond SL. Management of cervical ribs and anomalous first ribs causing neurogenic thoracic outlet syndrome. $\mathbf{J}$ Vasc Surg 2002;36:51-6.

[22]. Nannapaneni R, Marks SM. Neurogenic thoracic outlet syndrome. Br J Neurosurg 2003; 17:144-8.

[23]. Durham JR, Yao JS, Pearce WH, Nuber GM, McCarthy WJ 3rd. Arterial injuries in the thoracic outlet syndrome. J Vasc Surg 1995;21:57- 69; discussion: 70.

[24]. Sanders RJ, et al: Diagnosis of thoracic outlet syndrome. J Vasc Surg 46:601-604, 2007.

[25]. Fields W, et al: Thoracic outlet syndrome: review and reference to stroke in a major league pitcher. Am J Neuroradiol 146:809-814, 1986.

[26]. Gooneratne IK, et al: Pearls and oysters: distal subclavian artery. A source of cerebral embolism. Neurology 73:e11-e12, 2009.

[27]. Naz I, et al: Cerebral embolism: distal subclavian disease as a rare etiology. J Pak Med Assoc 56:186-188, 2006.

[28]. Gelabert HA, Machleder HI. Diagnosis and management of arterial compression at the thoracic outlet. Ann Vasc Surg 1997; 11:359-6614.

[29]. Schein CJ, Haimovici H, Young H. Arterial thrombosis associated with cervical ribs: Surgical considerations. Surgery 1956; 40:428-43.

[30]. Adson AW: Surgical treatment for symptoms produced by cervical ribs and the scalenus anticus muscle. Surg Gynecol Obstet 85:687-700, 1947.

[31]. Hugl B, et al: Unusual etiology of upper extremity ischemia in a sclero- derma patient: thoracic outlet syndrome with arterial embolization. J Vasc Surg 45:1259-1261, 2007.

[32]. Fields W, et al: Thoracic outlet syndrome: review and reference to stroke in a major league pitcher. Am J Neuroradiol 146:809-814, 1986.

[33]. Gooneratne IK, et al: Pearls and oysters: distal subclavian artery. A source of cerebral embolism. Neurology 73:e11-e12, 2009.

[34]. Naz I, et al: Cerebral embolism: distal subclavian disease as a rare etiology. J Pak Med Assoc 56:186-188, 2006. 